\title{
Long noncoding RNA Meg3 sponges miR-708 to inhibit intestinal tumorigenesis via SOCS3-repressed cancer stem cells growth
}

\author{
Shuo Zhang ${ }^{1,3}$, Wei-Wei $\mathrm{Ji}^{2,3}$, Wei Wei ${ }^{2}$, Li-Xing Zhan ${ }^{2}$ and Xuan Huang $\mathbb{1 D}^{1 凶}$ \\ (c) The Author(s) 2021
}

BACKGROUND: Colorectal cancer (CRC) remains the most common gastrointestinal cancer and a leading cause of cancer deaths worldwide, with most showing pathologies indicating the malignant transformation of early stage intestinal stem cells. The long non-coding RNA Meg3, which functions as a tumor suppressor, has been reported to be abnormal in multiple tumorigenesis events; however, the underlying mechanism by which Meg3 contributes to the malignant proliferation of colonic stem cells remains unclear.

METHODS: We analyzed the expression levels of Meg3, miR-708, and SOCS3 in samples from Apc loss-of-function (Apc ${ }^{\mathrm{min}}$ ) mice and patients with CRC, particularly in colonic crypt cells. $A p c^{\min }$ mice and AMO/DSS-induced mice model (in vivo) and organoid culture system (in vitro) were used to explore the effect of the Meg3/miR-708/SOCS3 axis on tumorigenesis in the colon. In vitro, we performed RNApull-down, RNA immunoprecipitation, and luciferase reporter assays using DLD1 and RKO cell lines.

FINDINGS: The Meg3/miR-708/SOCS3 signaling axis plays a critical role in the early stage of CRC development. Our data showed Meg3 levels negatively correlate with miR-708 levels both in clinical samples and in the $A p c^{\text {min }}$ mouse model, which indicated that Meg3 acts as a competitive endogenous RNA (ceRNA) of miR-708. Then, miR-708 served as an oncogene, inducing neoplasia in both $A p c^{m i n}$ mice and cultured colonic organoids. Put together, miR-708 appears to promote malignant proliferation of colonic stem cells by targeting SOCS3/STAT3 signaling.

INTERPRETATION: These data revealed that Meg3 sponges miR-708 to inhibit CRC development via SOCS3-mediated repression of the malignant proliferation of colonic stem cells. The Meg3/miR-708/SOCS3 signaling axis provides potential targets for the diagnosis and treatment of CRC, particularly early stage CRC.

Cell Death and Disease (2022)13:25; https://doi.org/10.1038/s41419-021-04470-5

\section{INTRODUCTION}

Colorectal cancer (CRC) remains the most common gastrointestinal cancer and a leading cause of cancer deaths worldwide [1-3]. Notably, the incidence and mortality of CRC continues to increase globally in recent decades [1-3]. Clinically, the most critical prognostic factor is the pathological stage of CRC at diagnosis [3]. Though the application and popularization of colonoscopy and the development of effective biomarkers has permitted remarkable progress in CRC diagnosis and therapy, the advanced CRC prognosis and diagnosis of early stage CRC remain major unmet medical needs. Therefore, it is urgent to explore the fundamental processes of CRC to identify more effective diagnostic and therapeutic targets for interventions against advanced colon polyps and early stage CRC.

Intestinal stem cells (ISCs), which are localized at the bottom of intestinal crypts, have been identified as central in CRC, such that malignant transforming events in ISCs contribute to the initiation of most intestinal cancers $[4,5]$. Lineage retracing has revealed that almost $75 \%$ of adenoma cells originate from Lgr5 ${ }^{+}$ISCs [6]. Consequently, it is necessary to investigate the underlying mechanism of malignant transformation of ISCs in early stage CRC.

Epigenetic changes, including alterations of noncoding RNA (ncRNA), are considered important contributors to tumorigenesis; epigenetic alterations in CRC occur earlier and more frequently than do genetic alterations [7, 8]. MicroRNAs (miRNAs) and long ncRNAs (IncRNAs) are the two most commonly studied ncRNAs in CRC. Notably, an increasing body of literature has identified miRNAs as clinically useful biomarkers for diagnosis and therapy of CRC; and emerging evidence suggests that dysregulated IncRNAs have functional effects on CRC pathogenesis, although the underlying mechanisms of most IncRNAs in CRC remain incompletely understood [7-9]. IncRNAs may serve as competitive endogenous RNAs (ceRNAs), "sponging up" miRNAs to deplete their level and thereby alter the expression of the miRNA's target genes [10]. Thus, the role of the IncRNA-microRNA axis in CRC has been the subject of recent attention, although the

\footnotetext{
${ }^{1}$ Department of Gastroenterology, The First Affiliated Hospital of Zhejiang Chinese Medical University, Hangzhou 310006, China. ${ }^{2}$ CAS Key Laboratory of Nutrition, Metabolism and Food Safety, Shanghai Institute For Nutritional Science, Shanghai Institutes for Biological Sciences, Chinese Academy of Sciences, Shanghai 200031 , China. ${ }^{3}$ These authors contributed equally: Shuo Zhang, Wei-Wei Ji. ${ }^{凶}$ email: huangxuan1976@163.com

Edited by Dr. George Calin
}

Received: 25 April 2021 Revised: 16 November 2021 Accepted: 10 December 2021

Published online: 21 December 2021 
specific contribution of this axis to the malignant transformation of ISCs in CRC is unknown. Efforts devoted to elucidating the underlying mechanisms are essential and warrant further exploration; such research is expected to provide promising biomarkers for the effective diagnosis and therapy of both early stage and advanced CRC.

A maternally expressed gene (Meg3) acts as a tumor suppressor in multiple cancers including CRC [11]. Meg3 levels are decreased in patients with CRC (compared to control patients); higher Meg3 levels have been shown to positively correlate with better overall survival and disease-free survival in CRC $[12,13]$. Accumulating evidence indicates that Meg3 is involved in cell proliferation, migration, invasion, and chemoresistance in CRC via "sponging" of mRNAs or miRNAs [13-15]. Meg3 depletion has been shown to strengthen stem-cell-like characteristics in diverse cell types, including lung cancer cells, mesenchymal stem cells, and germline stem cells $[16,17]$. However, the effects of $M e g 3$ in early stage CRC and malignantly transformed ISCs remain unclear.

Herein, we demonstrated a sponging role for Meg3 in the miR708/SOCS3 axis, affecting the colonic stemness of early stage CRC. Firstly, we surveyed the miR-omes of colonic crypt cells with different $M e g 3$ expression profiles using $A p c^{\min }$ mice (a strain that harbors a germ-line mutation in Apc, the mouse homolog of the human APC gene, which renders the animals prone to developing (RC); this work revealed that miR-708 is an important component in Meg3-related colonic stemness. Both in a mouse model and in the clinic, higher miR-708 levels indicated progression to later stages of CRC and poorer overall survival. In addition, Meg3 level negatively correlated with miR-708 level in CRC. Functionally, miR708 is capable of promoting colonic adenoma development in vivo and accelerated colonic organoid and CRC cell growth in vitro. Mechanistically, Meg3 sponged miR-708 to hijack organoid and CRC cell growth through SOCS3-induced growth inhibition. Notably, the sponging correlation among Meg3, miR-708, and SOCS3 were present in a colonic adenoma mouse model and in clinical CRC tissues. Collectively, the current study revealed the role of the Meg3/miR-708/SOCS3 axis in determining colonic stemness in early stage CRC, demonstrating that targeting the combination of Meg3 and miR-708 might represent a diagnostic and therapeutic strategy in early stage CRC.

\section{MATERIALS AND METHODS \\ Mice and mouse model}

All animals were bred, maintained, and used in accordance with the guidelines of the Institutional Animal Care and Use Committee of the Shanghai Institute of Nutrition and Health (SINH) (Shanghai, China). All mice were housed under specific-pathogen-free conditions in laboratories maintained at $23 \pm 3{ }^{\circ} \mathrm{C}$ with a relative humidity of $35 \% \pm 5 \%$ and a $12-\mathrm{h} /$ 12-h dark/light cycle. Throughout the study, animals were provided with free access to a standard diet (Shanghai Laboratory Animal Co., Ltd., Shanghai, China) and drinking water, except as noted below.

To generate miR-708 ${ }^{-1-}$ mice, the Sanger MirKO ES cell line miR-708 was microinjected into C57BL/6 mice at Shanghai Model Organisms (Shanghai, China); resulting male chimeric mice were crossed with $\mathrm{C} 57 \mathrm{BL} / 6$ females to generate heterozygous mice, which then were crossed to generate miR$708^{+1+}$ (wild-type, WT) and $m i R-708^{-1-}$ (knockout, KO) mice. WT and KO mice were co-housed until 4 weeks of age.

For the $A p c^{\text {min }}$ mouse model, WT or KO mice ( 8 weeks old, female) were crossed with $A p c^{\text {min }}$ mice (8-week-old male) to generate $A p c^{\text {min }}$ miR-708 $^{+/+}$ (wild-type) mice or $A p c^{\min } \operatorname{miR-708^{-1-}}$ mice. Male mice were used in colonic crypt isolation (from 8-week-old animals) and for the development of early stage CRC.

For the azoxymethane/dextran sulfate sodium (AOM/DSS)-induced colorectal cancer model, the mice were treated as previously described [18]. Briefly, mice (6-8-week-old-male) were injected subcutaneously with AOM $(10 \mathrm{mg} / \mathrm{kg})$ on Day-5, and then provided with free access to 3\% DSS (160110, MW = 36-50 kD; MP Biomedicals, Santa Ana, CA, USA) in the drinking water for 5 days starting on Day 0 (i.e., starting 5 days after administration of AOM). After the 5 th day on $3 \%$ DSS, the animals were shifted back to standard (unadulterated) drinking water. DSS treatment was repeated for 3 cycles every 16 days. To increase Meg 3 levels or decrease SOCS3 levels in colon tissues, Meg3 adenovirus (AdvMeg3), SOCS3 interference adenovirus (Adv-SOCS3i), or control adenovirus was administered to mice (5-week-old-male) by rectal instillation (per injection/7days).

All animal experiments were approved by the Institutional Animal Care and Use Committee of the Shanghai Institutes for Biological Sciences, Chinese Academy of Sciences (Shanghai, China).

\section{Human cohort}

This study was approved by the Institute Research Ethics Committee of the First Affiliated Hospital of Zhejiang Chinese Medical University. Two validated cohorts of patients with CRC were enrolled in this study. For each cohort, patients must have been pathologically confirmed with CRC. Tumor specimens from these patients were obtained at the time of surgical resection before therapy. Paired samples (tumor and adjacent normal tissue), embedded by formalin-fixed paraffin, were obtained from these patients who underwent operations and matched all clinicopathologic variables.

\section{Colonic crypt isolation and organoid culture}

Following euthanasia of 8- to 10-week-old male AOM/DSS mice, the entire colons were recovered, everted, and rinsed 5 times with ice-cold phosphate-buffered saline (PBS) supplemented with $2 \%$ (vol/vol) penicillin and streptomycin. Colonic crypts were isolated by immersing the everted and rinsed colons in chilled $\left(0-4{ }^{\circ} \mathrm{C}\right)$ Cell Recovery Solution buffer (Corning Cat. 354253; Corning, NY, USA) for 30-40 min before passage through $70-\mu \mathrm{m}$ strainers to filter the resulting cell suspension. The suspended cells then were rinsed 3-4 times with basic cell culture medium (DMEM/F12; Gibco $^{T M}$, Waltham, MA, USA) supplemented with $1 \%$ (vol/vol) penicillin and streptomycin $\left(\mathrm{Gibco}^{\mathrm{TM}}\right), 1 \%$ GlutaMAX $\left(\mathrm{Gibco}^{\mathrm{TM}}\right)$, and $1 \%$ HEPES buffer (4--1-piperazineethanesulfonic acid; $\mathrm{Gibco}^{\mathrm{TM}}$ ). The isolated colonic crypts were resuspended in Matrigel (BD Cat. 354230; BD) and plated in pre-warmed $\left(37^{\circ} \mathrm{C}\right) 24$-well culture plates, which then were incubated in a cell culture incubator (at $37^{\circ} \mathrm{C}$, in a humidified atmosphere with $5 \% \mathrm{CO}_{2}$ ) for 10 min after seeding. Colon organoid growth medium (consisting of a 1:1 mixture of DMEM/F12:L-Wnt 3 A supernatant (see below) supplemented with $20 \%$ (vol/vol) fetal bovine serum (FBS; Bl), 1\% (vol/vol) penicillin and streptomycin, $500 \mathrm{ng} / \mathrm{mL}$ R-Spondin 1 (Cat. 3474-RS, R\&D), $50 \mathrm{ng} / \mathrm{mL}$ epidermal growth factor (EGF; Cat. 50482-M01H; Sino Biological Co., Ltd., China), $100 \mathrm{ng} / \mathrm{mL}$ Noggin (Cat. 250-38-20, Peprotech), $10 \mu \mathrm{m}$ Y-27632 (Cat. 1254; Tocris)) was distributed to the culture plates at $500 \mu \mathrm{L} /$ well; the day of the addition of this growth medium constituted the nominal Day 0 for organic growth. After culture for the indicated times, organoids were subjected to western blot or quantitative reverse transcription-polymerase chain reaction ( $q$ RT-PCR) analysis.

For passaging, organoids were washed with cold PBS. After centrifugation at $200 \times g$ for $2-3 \mathrm{~min}$, the organoids were resuspended with Matrigel $(50 \mu \mathrm{L} /$ well) and then plated. For miR-708 agomir, miR-708 antagomir, or Meg3 interference studies, the agomir, antagomir, or adenovirus was added to the growth medium at Day One.

The L-Wnt $3 \mathrm{~A}$ cell line was purchased from Shanghai Fuxiang Biotechnology Co., Ltd. and cultured in growth medium (DMEM supplemented with $10 \%$ FBS and $1 \%$ (vol/vol) penicillin and streptomycin) to $80-90 \%$ cell confluence; the resulting conditioned medium was collected every three days for a total of three times and filtered through $0.22-\mu \mathrm{m}$ filters. The diameter and the number of organoids were measured by ImageJ.

\section{Cell lines and cell culture}

The colorectal cancer cell lines (DLD1 and RKO) and the IMEC cell line were obtained from our laboratory collection. All of the above cell lines were cultured with DMEM medium (Life Technologies) supplemented with $10 \%$ FBS and $1 \%$ penicillin/streptomycin and incubated at $37{ }^{\circ} \mathrm{C}$ in a humidified atmosphere with $5 \% \mathrm{CO}_{2}$.

\section{Cell proliferation assay}

DLD1 or RKO cells (4000/well) were plated in 96-well plates in triplicate. The Cell Counting Kit 8 (CCK8) (Abcam) was used to measure cell proliferation. We performed this assay according to the manufacturer's protocols. 
Table 1. qRT-PCR primer sequences.

\begin{tabular}{ll} 
Gene & Forward primer $\mathbf{5}^{\prime} \mathbf{- 3}^{\prime}$ \\
\hline hSOCS3 & CCTGCGCCTCAAGACCTTC \\
mSOCS3 & TGCGCCTCAAGACCTTCAG \\
\hline hMeg3 & CATCTACACCTCACGAGGG \\
mMeg3 & TCCTGGATTAGGCCAAAGC \\
\hline MiR-708 & CGTCAAGGAGCTTACAATCT \\
mGAPDH & AGGTCGGTGTGACGGATTTG \\
hGAPDH & CTGGGCTACACTGAGCACC \\
\hline
\end{tabular}

Reverse primer $\mathbf{5}^{\prime} \mathbf{- 3}^{\prime}$
GTCACTGCGCTCCAGTAGAA
GCTCCAGTAGAATCCGCTCTC
ATCCTTTGCCATCCTGGTC
AGCCTATTTGAGAAGCTGGT
CAGTGCGTGTCGTGGAGT
TGTAGACCATGTAGTTGAGGTCA
AAGTGGTCGTTGAGGGCAATG

\section{Western blot}

Western blot assays were performed as described previously [19]. Immunoblotting in the present study employed antibodies (purchased from Cell Signaling Technology, Boston, MA, USA) against SOCS3, Stat3, pStat3(Tyr705), Hsp90, and the housekeeping protein glyceraldehyde phosphate dehydrogenase (GAPDH); GAPDH and Hsp90 were used as a loading control.

\section{RNA extraction and qRT-PCR}

RNA extraction and qRT-PCR were performed as described previously [19]. The primers used in this study are shown in Table 1.

\section{Hematoxylin and eosin (HE) staining, immunohistochemistry (IHC), immunofluorescence (IF), and RNA-fluorescence in situ hybridization (RNA-FISH)}

HE staining, IHC, and IF were performed as described previously $[19,20]$ IHC employed the following reagents: anti-Ki67 antibody (Novus Cat. NBP1-40684), anti-SOCS3, and anti-p-Stat3 antibodies (Cell Signaling Technology). IF employed the following reagent: anti-p-Stat3 antibody (Cell Signaling Technology).

For the RNA-FISH assay, the colonic tumor tissues from $A p c^{+/ m i n}$ mice were recovered at necropsy, washed with PBS, fixed in 4\% PFA buffer (paraformaldehyde diluted in PBS that had been pre-treated with Diethylpyrocarbonate). The fixed tissues then were embedded in paraffin. After sectioning with an Ultracut microtome (Leica, Bannockburn, IL, USA), the embedded tissues were dehydrated and digested with Protein $\mathrm{K}$, prehybridized for $1 \mathrm{~h}$, and hybridized with miR-708 probe overnight at $37^{\circ} \mathrm{C}$. The miR-708 probe consisted of a 6-carboxyfluorescein (FAM)-labeled oligonucleotide with the sequence $5^{\prime}$-FAM-GGGUCGAUCUAACAUUCGAG GAA-FAM-3'; the oligo was purchased from Guangzhou Ribobio Co., Ltd. (Guangzhou, China). After incubation with 4',6-diamidino-2-phenylindole (DAPI) staining buffer for $10 \mathrm{~min}$, the sections were mounted and then examined with an Olympus IX81 fluorescent microscope.

\section{RNA pull-down assay}

The RNA pull-down assay was performed as described previously [21]. miR-708 or Meg3, labeled with biotin, was transfected into cells for $24 \mathrm{~h}$, followed by incubation of the cell lysates with M-280 streptavidin magnetic beads (Sigma). Finally, the Meg3 or miR-708 level was measured by qRT-PCR.

\section{Luciferase reporter assay}

We performed the luciferase reporter assay as described previously. Briefly, the DNA sequences corresponding to the $3^{\prime}$-UTR of the SOCS3 or Meg3 transcript, which contain putative miR-708 binding sites, were amplified and cloned into the pGL3 vector. Mutation of the sequences at the specific putative binding sites was performed using the Quick Mutation ${ }^{\text {m }}$ SiteDirected Mutagenesis Kit (Beyotime Biotechnology, China). For the Meg3related luciferase reporter assay, the Meg3-WT or Mut luciferase vector and the miR-708 antagomir were co-transfected into DLD cells for $24 \mathrm{~h}$; separately, the Meg3-WT or Mut luciferase vector was transfected into RKO cells (which exhibit constitutive miR-708 expression) for $24 \mathrm{~h}$. For the SOCS3-related luciferase reporter assay, the SOCS3 3'UTR-WT or Mut vector was co-transfected with either the control agomir or the miR-708 agomir into cells pre-treated with Adv-Meg3 for $24 \mathrm{~h}$; separately, the SOCS3 $3^{\prime} \mathrm{UTR}$ WT or Mut vector was co-transfected with either the control antagomir or miR-708 antagomir into cells pre-treated with Adv-Meg3i for $24 \mathrm{~h}$. Following transfection, luciferase activity was assessed using a dual-luciferase reporter assay system (Promega, USA); and the relative luciferase activity was normalized to that of Renilla luciferase activity.

\section{RNA immunoprecipitation (RIP) assay}

The RIP assay was performed using the Magna RIP Kit (Millipore) according to the manufacturer's protocols. Briefly, cells that had been transfected with the miR-708 antagomir, miR-708 WT, or miR-708 Mut for $24 \mathrm{~h}$ were subjected to the RIP assay; cell lysates then were incubated with antibodies against IgG or AGO2. Meg3 levels in the RIP products were detected using qRT-PCR.

\section{Statistical analysis and bioinformatics analysis}

miR-708 targets and the paired sites of Meg3 were predicted using the online websites miRbase (http://www.mirbase.org), Targetscan (http:// www.targetscan.org), and miRDB (http://www.miRdb.org). CRC datasets were obtained from GEO datasets. Experimental data are presented as the mean $\pm S . D$ and were analyzed by unpaired, two-tailed Student's $t$ tests; analyses were conducted using Prism 6.01 software (GraphPad; San Diego, CA). $p$ Values of less than 0.05 were considered significant.

\section{Generation and administration of recombinant}

Adenoviruses Recombinant adenoviruses expressing Meg3 (Adv-Meg3), SOCS3 (Adv-SOCS3), and Interfering AF6 (Adv-Meg3i), SOCS3 (Adv-SOCS3i) were constructed using the AdMax system (OBiO Technology) according to the manufacturer's instructions. Purified high-titer stocks of amplified recombinant adenoviruses were diluted in PBS and administered at a dose of $1 \times 10^{7}$ plaque-forming units (PFU)/well in 12-well plates or injected at a dose of $1 \times 10^{9} \mathrm{PFU} /$ mouse through coloclysis. Injection of the adenoviruses above or control viruses did not affect food consumption compared with that of control-treated animals.

\section{Flow cytometry}

Organoids were dissociated into a single with Accutase, and large clusters were removed with a 40 Micrometer Cell Strainer (Corning). The cells were washed with cold PBS and then fixed with Fixation/Permeabilization buffer (Biogems) at $4{ }^{\circ} \mathrm{C}$ for the night. The Cells were incubated with the LGR5 antibody(Abcam) at $1 / 25$ dilution at $37^{\circ} \mathrm{C}$ for $60 \mathrm{~m}$. Then Cells were incubated with the second antibody at room temperature for $30 \mathrm{~m}$. The cells were suspended with flow cytometry staining buffer, then assayed using a Flow cytometer.

\section{Soft agar assay}

Totally, $1 \times 10^{3}$ cells were inoculated into $0.35 \%$ agar containing $1 \times$ medium and seeded in each well of a 6-well plate containing $0.6 \%$ agar in $1 \times$ medium. Cells were grown at $37^{\circ} \mathrm{C}$ for 21 days. Cells were fixed with $4 \%$ paraformaldehyde and stained using $0.1 \%$ crystal violet. The cells were imaged and quantified with ImageJ.

\section{RESULTS}

Meg3 levels negatively correlate with miR-708 levels in colonic crypts of $\mathrm{Apc}^{\mathrm{min}}$ mice

Colon cancer might arise from a rare population of cells with stemcell-like properties. Previous work using the novel $\mathrm{Cre}^{+} \mathrm{Apc}^{+/+}$ mouse model showed that the acute and wide-spread loss of Apc in the colon results in crypt hyperplasia, demonstrating that Apcdeficient cells maintain a "crypt progenitor-like" phenotype [22]. 


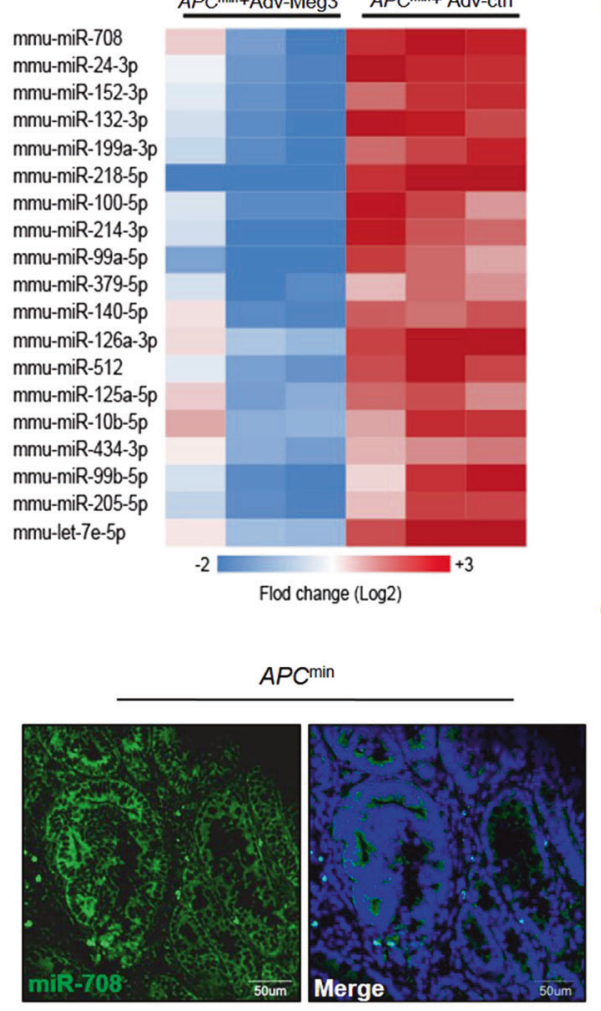

b

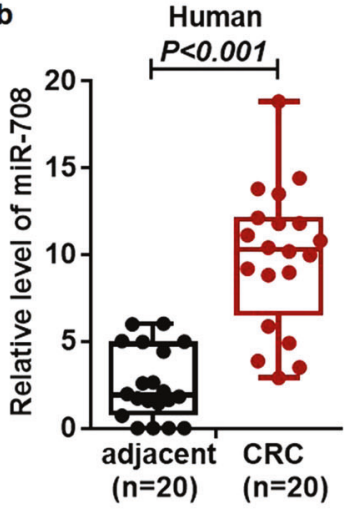

C

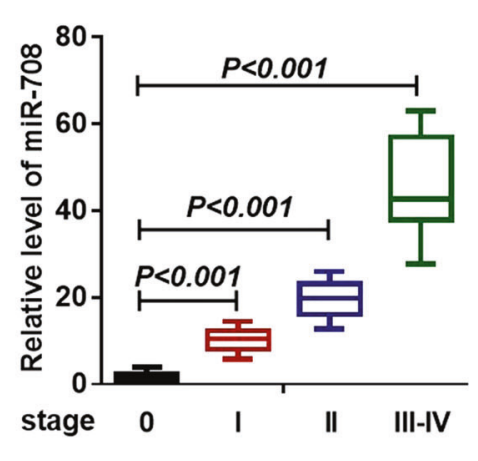

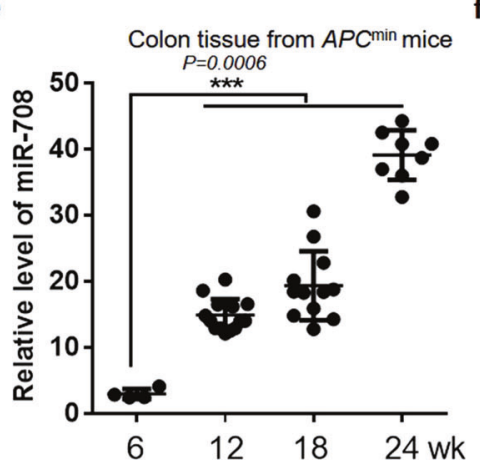

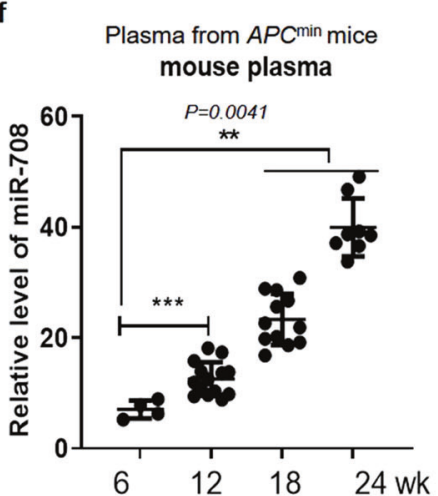

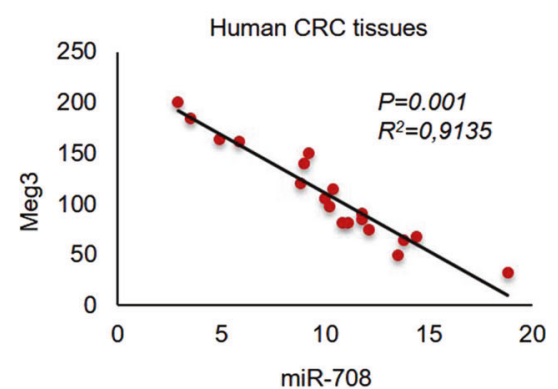

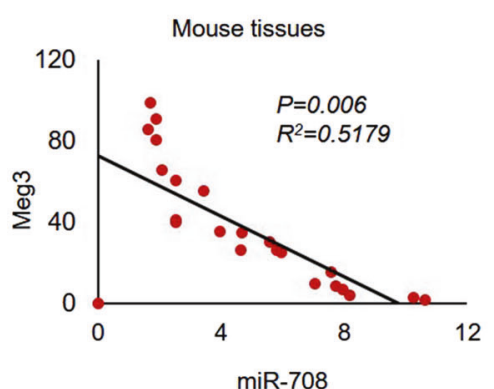

i

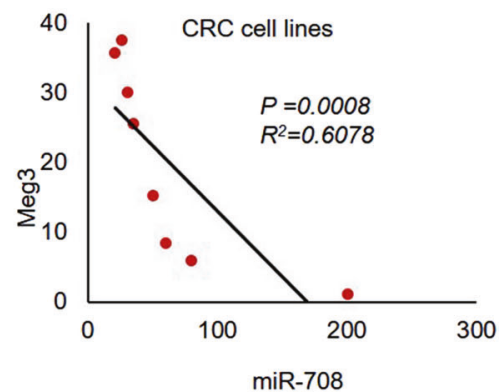

Fig. 1 The level of Meg3 negatively correlates with that of $\mathbf{m i R}-\mathbf{7 0 8}$ in colonic tumorigenesis. a A heat map showing miRs with significant accumulation (fold change $>2, p<0.05$ ) in colonic crypts from Apc ${ }^{\min }$ mice (12 weeks old) administered (rectal instillation) with the Meg3overexpressing adenovirus (Adv-Meg3) or control adenovirus (Adv-ctrl). b The relative expression of miR-708 in 20 pairs of CRC samples and adjacent normal samples by qPCR analyses. c The relative expression of miR-708 in tissues with different disease stages of CRC from TCGA dataset. d RNA-FISH assay was performed to detect the level and location of miR-708 in colonic adenoma from $A p c^{\mathrm{min}}$ mouse (22 weeks old). e, f The relative expression of miR-708 in colonic tissues (e) and plasma (f) from Apc ${ }^{\min }$ mice of the indicated ages. g-i Plots showing the correlation between miR-708 and Meg3 levels in human CRC tissues $(\mathbf{g}), A p c^{\text {min }}$ mouse colonic tissues (h), and CRC cell lines (i). The results are presented as the mean \pm S.D. in panels $(\mathbf{b}, \mathbf{c}, \mathbf{e}, \mathbf{f}) .{ }^{* *} p<0.01,{ }^{* *} p<0.001$ (non-paired two-tailed Student's $t$ test).

We suspected that abnormal expression of the IncRNA Meg3 might play a role in stem cell proliferation/transformation. We confirmed our hypothesis using colonic crypts isolated from $A p C^{\text {min }}$ mice, a typical colonic adenoma mouse model [23], and WT mice, showing that $M e g 3$ accumulates to markedly lower levels in the crypts of colonic tumor samples compared to those from WT mice (Supplementary Fig. 1a). To understand the ceRNA role of Meg3 in early stage CRC, $A p c^{\text {min }}$ mice were injected with adenovirus-Meg3 (Adv-Meg3) or a control virus (Adv-ctrl), followed by a miR-omes microarray assay. As shown in the heat map in Fig. $1 \mathrm{a}$, the levels of multiple miRNAs were significantly decreased (fold change $\geq 2, p$ value $\leq 0.05$ ) in Adv-Meg3- transformed (Meg3-overexpressing) colonic crypts compared to those transformed with the control virus. Among these altered miRNAs, miR-708 was among the top three miRNAs with decreased accumulation, suggesting that this miRNA may contribute to CRC oncogenesis. miR-708-5p has been widely studied for its role as an oncogenic miRNA [24]. Therefore, we next assessed miR-708 expression in 20 pairs of clinical samples, each consisting of $\mathrm{CRC}$ and adjacent normal (control) tissues. We found that miR-708 accumulated significantly higher levels in CRC tissues compared to the adjacent normal tissues (Fig. 1b). To clarify this observation, the miR-708 levels in CRC tissues from patients with different pathological stages (normal and stages I, II, and III-IV) 
were further analyzed, revealing that miR-708 levels exhibited a positive correlation with CRC malignant progression (Fig. 1C). We used the $A p C^{\text {min }}$ mouse model to obtain samples from animals that spontaneously developed intestinal adenomas. An RNA-FISH assay was performed to detect the expression and location of miR-708 in the resulting colonic adenomas. We observed that miR-708 localized primarily in the cell cytoplasm and was detected at a higher level in adenomas compared to the adjacent tissues (Fig. 1d). In addition, miR-708 levels were measured in $A p C^{\min }$ mice of various ages; the data showed that miR-708 levels increased in colonic tissues and plasma in a time-dependent manner (Fig. 1e, f).

Given that Meg3 functions as a critical tumor suppressor in cancer $[24,25]$, the GEO datasets were analyzed to assess Meg3 expression in CRC. We found that Meg3 levels were decreased in tumor tissues from CRC patients (Supplementary Fig. 1b), as well as in AOM/DSS-induced CRC mice (Supplementary Fig. 1c), compared to the corresponding control tissues. Consequently, we quantified Meg3 and miR-708 levels in CRC tissues and CRC cells. Correlation analysis of the resulting data revealed that Meg3 levels negatively correlated with those of miR-708 in CRC (Fig. 1g-i).

Together, our data demonstrated that Meg3 levels were negatively correlated with miR-708 levels; notably, higher miR708 levels indicated progression to later stages of CRC. Furthermore, these results suggest that miR-708 may be a target of Meg3, a IncRNA that is responsible for crypt hyperplasia.

\section{miR-708 deficiency decreases the occurrence of colonic adenoma and inhibits colonic organoid growth}

miR-708 has been identified as an oncogene in multiple CRC cell lines $[25,26]$, but evidence for the biological role of miR-708 in vivo remains limited. Therefore, we generated miR-708-knockout $A p c^{\text {min }}$ mice $\left(A p c^{\text {min }} \mathrm{miR}^{-708^{-/-}}\right)$to investigate the role of miR-708 in early stage CRC. qRT-PCR analysis of $A p c^{\text {min }}$ miR-708 ${ }^{-1-}$ mice suggested that miR-708 was depleted successfully in the colon of these animals (Fig. 2a). As shown in Fig. 2b, Apc ${ }^{\text {min }}$ miR$708^{-1-}$ mice exhibited fewer and smaller adenoma tissues compared to $A p C^{\mathrm{min}}$ mice, suggesting miR-708 depletion significantly $(p<0.05)$ suppresses colon tumor development in vivo (Fig. 2b). It was reported that the intestinal adenomas was appear from 4 weeks in $A p c^{m i n}$ mice. Thus, we scarified the $A p c^{\text {min }}$ miR- $^{\text {mice }}$ $708^{+/+}$(wild-type) mice or $A p c^{\min } \mathrm{miR}^{-708^{-1-}}$ mice at 4 weeks and IHC staining colon tissues for analysis its pathological state. As shown in Fig S2e, there was almost no adenoma burden in the colon of $A p C^{\mathrm{min}} \mathrm{miR}-708^{-1-}(0 / 6)$ mice, but we do observe some intestinal adenoma in $A p c^{\text {min }}$ miR-708 ${ }^{+/+}(3 / 7)$ mice at this stage. Subsequently, we performed staining using $H \& E$ and $I H C$. The results from the H\&E staining assay suggested that miR-708 deficiency attenuated the malignancy of tumor cells, and the data from IHC staining for Ki67 showed that the level of Ki67 was decreased in colonic tissues from $A p c^{\text {min }} \mathrm{miR}_{-108^{-1-}}$ mice (compared to that in colonic tissues of $A p c^{\min }$ animals), indicating that loss of miR-708 blocked the over-proliferation of colonic cells typically seen in $A p c^{\mathrm{min}}$ mice (Fig. 2c, Fig S2f). Assessment of the survival of $A p c^{\min }$ mice using Kaplan-Meier analysis revealed that $A p c^{\text {min }}$ miR-708 ${ }^{-1-}$ mice exhibited better overall survival than $A p c^{\text {min }}$ mice (Fig. 2d). In addition, to further characterize the effect of miR-708 in early stage CRC, we employed the AOM/DSSinduced CRC model. The results of tumor development, histology, and Ki67 staining in tissues from mice of this model were coincident with those in tissues from $A p c^{\text {min }}$ mice (Supplementary Fig. 2a-f). Together, these data confirmed that miR-708 has an oncogenic function in early stage CRC.

We established IMCE-Ras cell lines, which contain the activated $\mathrm{v}$-H-ras gene (Fig. S1d). We performed a soft agar assay with the treatment of miR-708 mimic or control mimic in IMCE-Ras cells. Mir-708 mimic treated IMCE-Ras cells have a more spindle-like appearance of transformed cells compared with control treatment (Fig. S1e). Next, to explore the effect of miR-708 in colonic stem cells, we employed a colonic stem cell-derived organoid culture system (Fig. S1f), in which colonic crypts from $A p C^{\text {min }}$ mice were isolated and then allowed to develop into colonic organoids in Matrigel; this culturing system permitted organoids to maintain physiological characteristics similar to those generated in vivo. We observed that miR-708 depletion inhibited organoid growth (Fig. 2e), a result consistent with the results obtained with colonic organoids derived from AOM/DSS-induced CRC mice (Supplementary Fig. 2d). Notably, the crypts from $A p c^{\min }$ mice readily developed organoids more frequently than did those from $A p c^{\mathrm{min}}$ miR-708 ${ }^{-1-}$ mice, and miR-708 deficiency attenuated the development of organoids after serial passage (Fig. 2f). In addition, our results from 3D- and 2D-culture systems further showed that forced expression of miR-708 promoted cell proliferation in the RKO and DLD1 CRC cell lines (Fig. 2g). To test the effect of miR-708 depletion, we designed a miR-708 antagomir system. As expected, miR-708 knockdown (using this antagomir) inhibited proliferation in both CRC cell lines (Supplementary Fig. 3a, b).

Taken together, these data suggested that miR-708 fosters early stage CRC development; this effect may be mediated by elevating the malignant proliferation of colonic stem cells.

\section{Meg3 sponges miR-708 to inhibit malignant proliferation of colonic stem cells in CRC}

Given that miR-708 levels are decreased in the presence of Meg3 in colonic crypt cells, we hypothesized that Meg3 acts as a ceRNA in regulating miR-708 levels, which led us to investigate the potential sponging relationship between $M e g 3$ and miR-708. To address the underlying mechanism, database prediction tools were used. Bioinformatics analyses identified putative Meg3binding sequences in miR-708 and showed that these putative binding sequences are conserved between humans and mice (Fig. 3a). Next, we constructed Meg3- and miR-708-WT or Mut plasmids based on these potential binding sequences; the plasmids were labeled with biotin and then transiently transfected into DLD1 and RKO cell lines (respectively), followed by the enrichment detection using an RNA pull-down assay. The results showed that miR-708 was enriched in Meg3-WT-pulled down RNAs, while Meg3-Mut failed to pull down miR-708 (Fig. 3b). Conversely, Meg3 was pulled down by miR-708-WT but not by miR-708-Mut (Fig. 3c). These data suggested that Meg3 specifically binds to miR-708 by base pairing. To further verify the Meg3-miR-708 interaction, a luciferase reporter assay was performed by co-transfecting CRC cell lines either with pGL3-Meg3-WT in combination with the miR-708 agomir or with pGL3-Meg3-Mut in combination with the miR-708 antagomir. We found that miR-708 negatively regulated luciferase activity only with Meg3-WT, and not with Meg3-Mut (Fig. 3d). Anti-AGO2 RIP assays also have been used to measure the endogenous interactions between a miRNA and its targeted sequences, given that $\mathrm{AGO} 2$ is critical for miRNA-induced RNA repression and degradation [27]. Therefore, we conducted an anti-AGO2 RIP assay in CRC cancer cells. The results showed that miR-708 counteracted the accumulation of Meg3, while a miR-708 antagomir had the opposing effect (Fig. 3e). Collectively, these results indicated the Meg3 interacts with $m i R-708$ and is involved in a miR708-mediated RNA silencing complex.

To further validate the negative correlation between Meg3 and miR-708 levels in CRC cells, we detected Meg3 levels in CRC cells with forced expression of miR-708 or that had been transfected with a miR-708 antagomir. In both cell lines, qRT-PCR analysis showed that miR-708 overexpression counteracted Meg3 accumulation in DLD1 and RKO cells, whereas introduction of the miR-708 antagomir led to Meg3 accumulation (Fig. 3f). Furthermore, in either $A p C^{\mathrm{min}}$ mice or AOM/DSS-treated mice, miR-708 deficiency elevated Meg3 levels in colonic tissues as well 


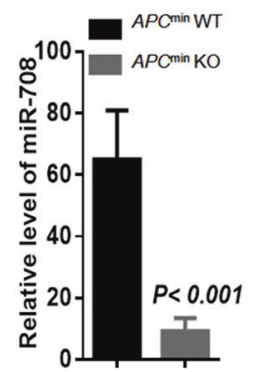

b

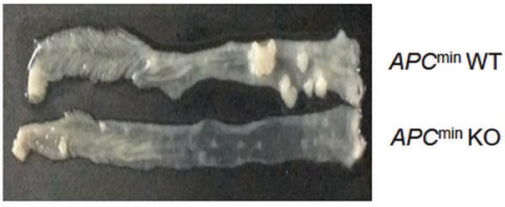

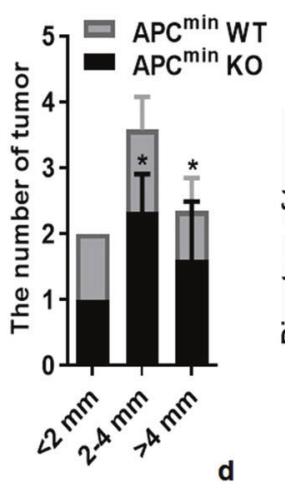

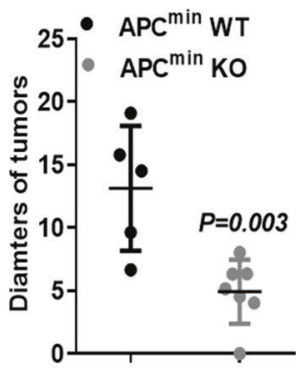

c

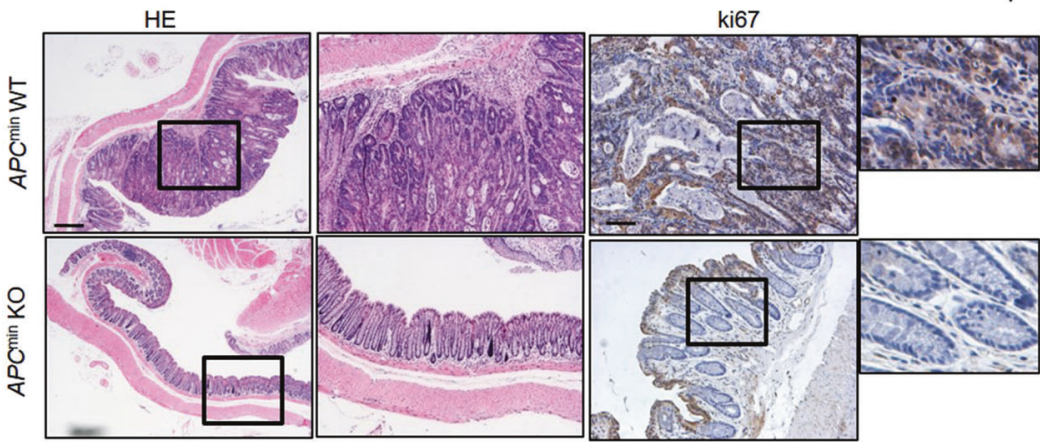

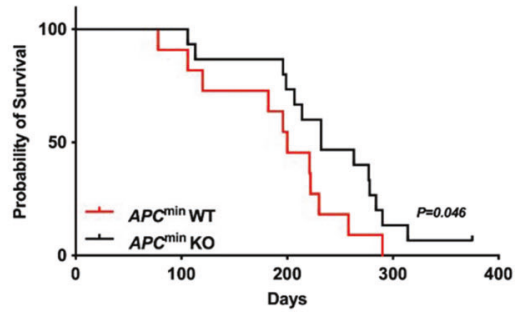

e

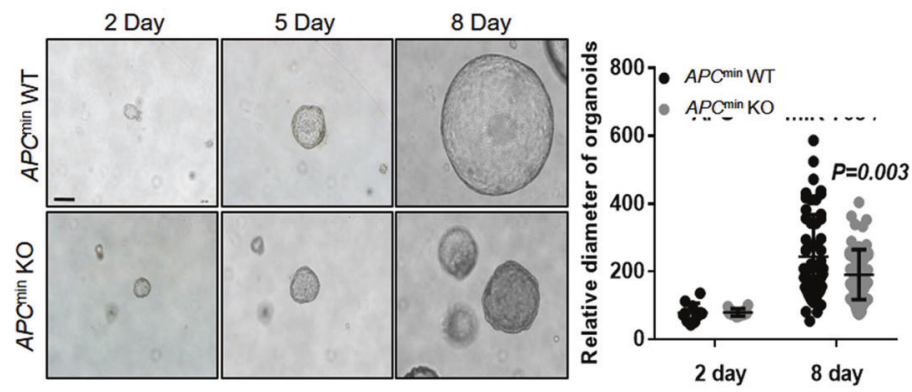

g
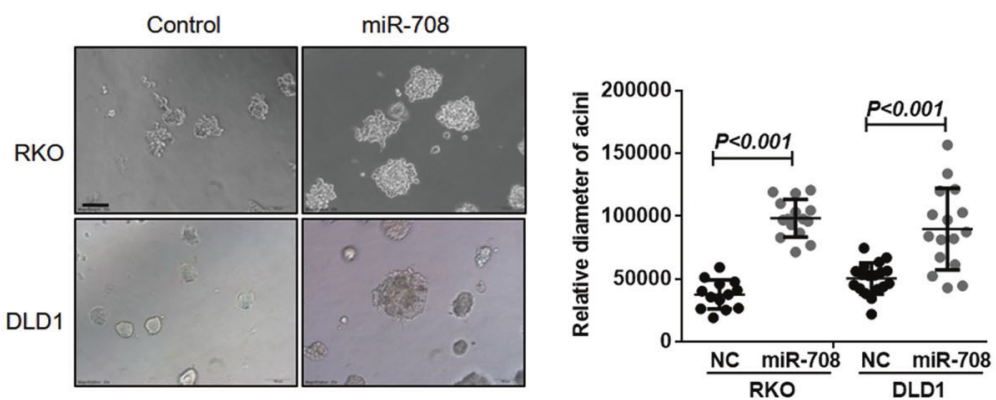

f

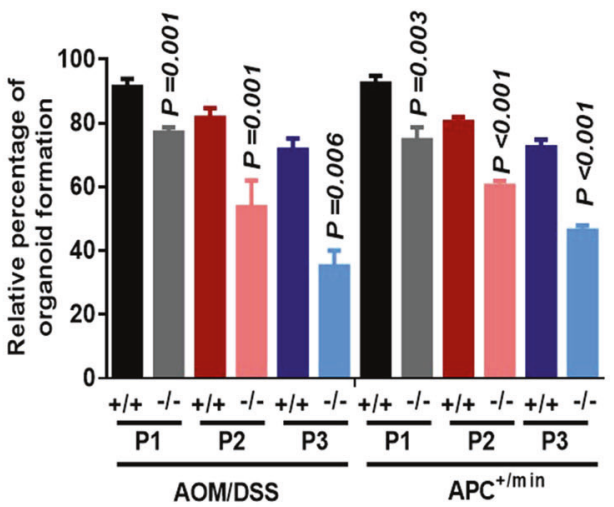

Fig. 2 miR-708 deficiency decreases colonic adenoma formation and inhibits colonic organoid growth. a-d $A p c^{\text {min }}$ sporadic colorectal cancer model. a The knockout effect of miR-708 in colon tissues from $A p c^{\mathrm{min}}$ mice. b Representative images of colonic tumors from $A p c^{\mathrm{min}}$ wild-type (WT) mice and $A p c^{\min }$ miR-708 KO (KO) mice. The plots show the statistics for tumor number ( $y$-axis) at different tumor diameters ( $x$-axis). c Representative images of HE staining (scale bar: $200 \mu \mathrm{m}$ ) and IHC staining for Ki67 (scale bar: $100 \mu \mathrm{m}$ ). d Kaplan-Meier analysis in WT and KO mice. e Representative images (scale bar: $100 \mu \mathrm{m}$ ) and quantification of organoid size. Organoids derived from the colonic crypts from $A p c^{\mathrm{min}}$ WT or $A p c^{\mathrm{min}}$ miR-708 KO mice were cultured for 8 days. $\mathbf{f}$ The statistics of the percentages of organoid formation after passage (P). Organoids were derived from AOM/DSS-induced colon cancer mice or $A p c^{\mathrm{min}}$ mice. g Representative images (scale bar: $\left.100 \mu \mathrm{m}\right)$ and quantification of the size of acinar spheres after 6 days of 3D-culture. RKO miR-708 and DLD1 miR-708 cells were infected with retrovirus and constitutively express miR-708. The results are presented as the mean \pm S.D. in panels $\left(\mathbf{a}, \mathbf{b}\right.$, and e-g). ${ }^{*} p<0.05$ (non-paired two-tailed Student's $t$ test) in panel (b). 
a

5' ugcaugugggagugggcuccuU 3' WT Meg3 (human)

3' GgGuCGAUCUAACAUUCGAgGAA 5' WT miR-708

5' CUUAAUUCAAAACCGgGCUCCUG 3' WT Meg3 (mouse)

5' UGCAUGUGgGAaGUGGCGAGGAA 3' mutant Meg3

3' GGGUCGAUCUAACAUUGCUCCUU 5' mutant miR-708

d
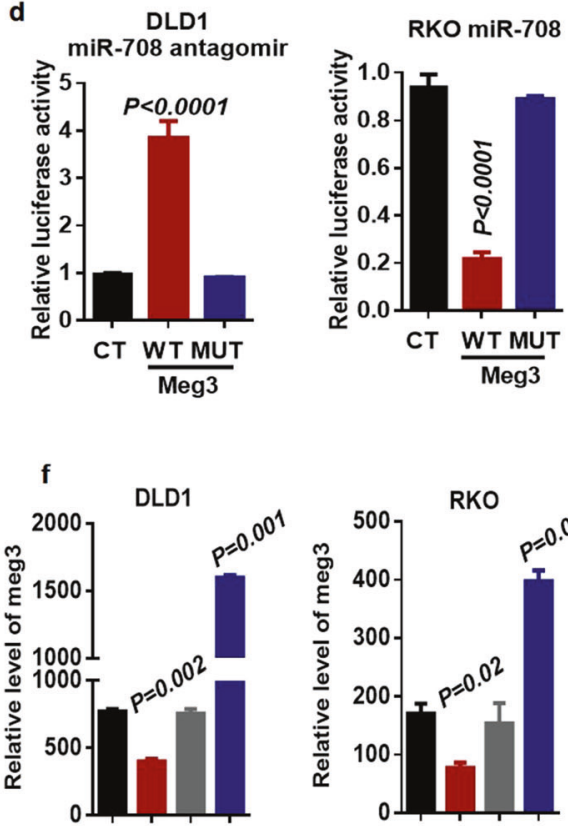

i

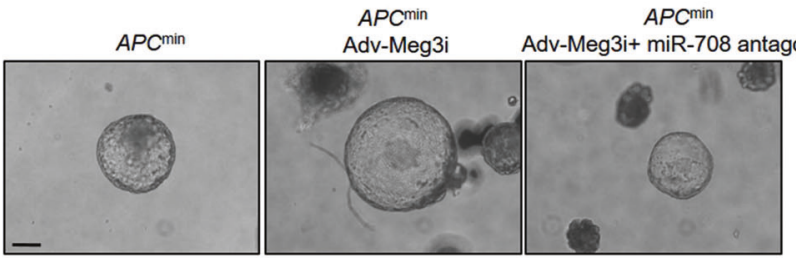

j

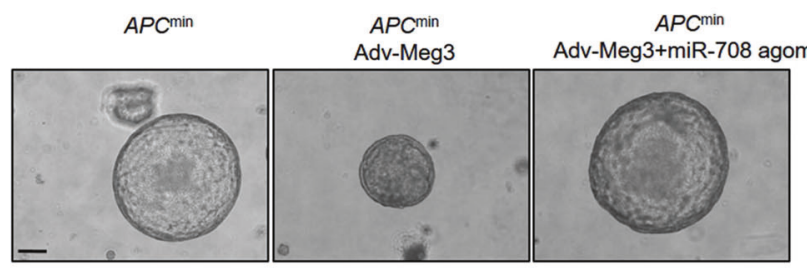

b

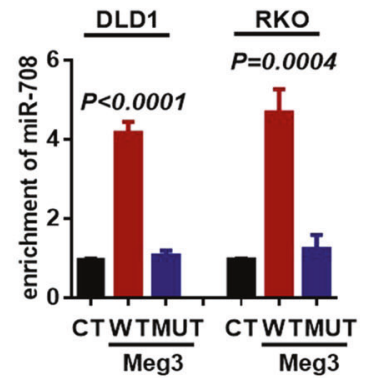

e
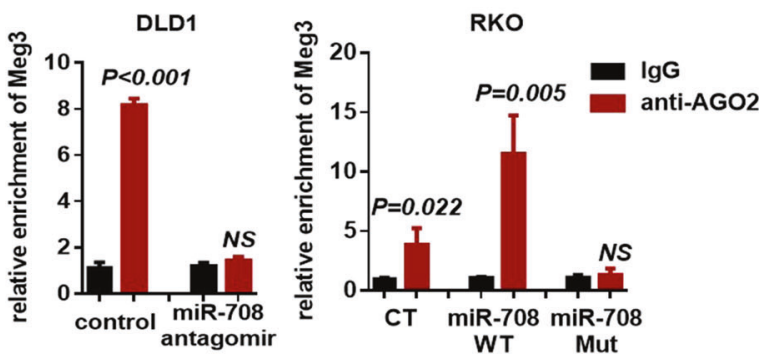

CRC tissue
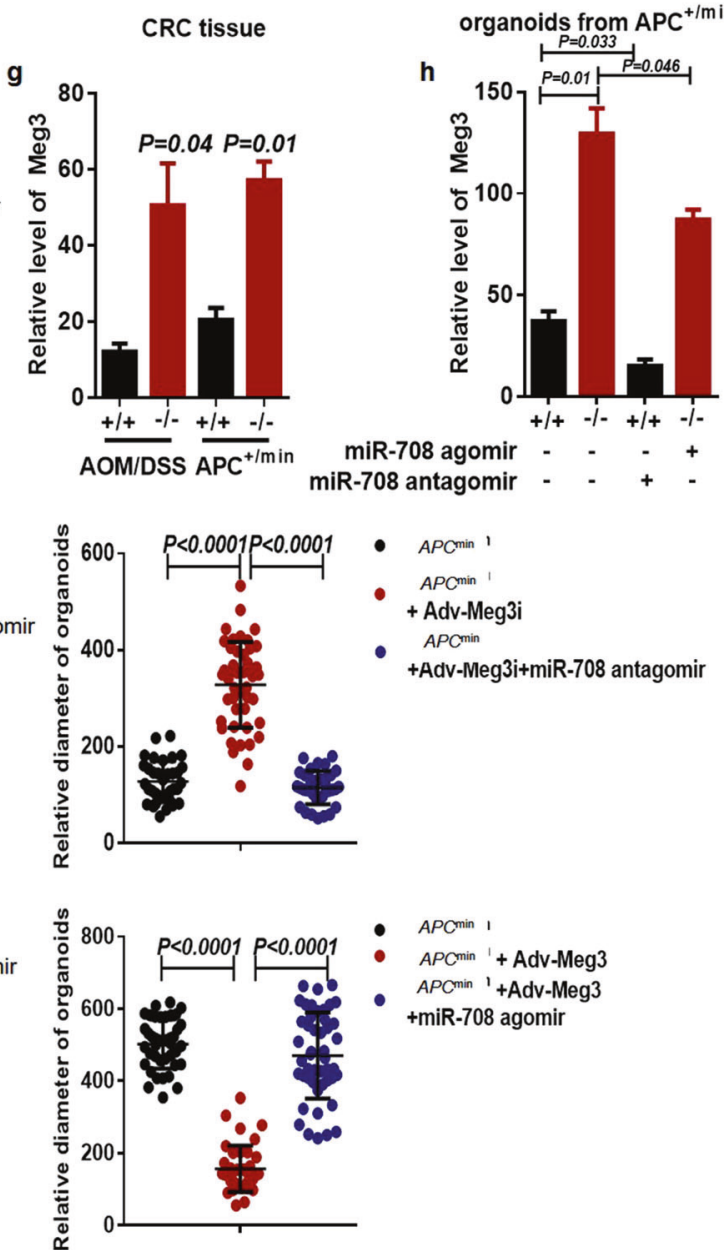

as in colonic crypt cells (Fig. $3 \mathrm{~g}, \mathrm{~h}$ ). We further explored the role of the Meg3-miR-708 axis in colonic stem cell growth using the organoid culture system and found that loss of Meg3 enhanced organoid growth, while overexpression of Meg3 suppressed colonic organoid growth (Fig. 3i, j). As expected, the miR-708 agomir rescued the Meg3-induced growth inhibition; consistent with that observation, the miR-708 antagomir counteracted the increased organoid growth seen with Meg3 deficiency (Fig. 3i, j). In addition, the data from the proliferation assay verified that Meg3 inhibited CRC cell proliferation, an effect that was reversed by miR-708 (Supplementary Fig. 4).

Together, these results demonstrated that Meg3 inhibits malignant proliferation of colonic stem cells and CRC cells via sponging of miR-708. 
Fig. 3 Meg3 sponges miR-708 to inhibit malignant proliferation of colonic stem cells in CRC. a Predicted base-pairing between miR-708 and Meg3 in human and mouse. Mutants (MUT) of miR-708 and Meg3 were generated by site-specific mutation of the paired bases. $\mathbf{b}$ DLD1 and RKO cell lysates were incubated with biotin-labeled Meg3 (WT and MUT types). RT-qPCR analyses were performed to detect miR-708 levels in the RNA pulldown obtained using biotin-labeled Meg3. c DLD1 and RKO cell lysates were incubated with biotin-labeled miR-708 (WT and MUT types). RT-qPCR analyses were performed to detect Meg3 levels in the RNA pulldown obtained using biotin-labeled miR-708. $\mathbf{d}$ Luciferase activities were measured in DLD1 and RKO cells. The cells were transfected with the indicated plasmids. e An AGO-RIP assay was used to measure Meg3 levels following miR-708 knockdown in DLD1 cells (left) or overexpression in RKO cells (right). $\mathbf{f}$ The relative levels of Meg3 were detected by qPCR in DLD1 and RKO cells constitutively expressing miR-708 or in DLD1 and RKO cells transfected with miR-708 antagomir. $\mathbf{g}$ RTqPCR analyses were performed to detect the relative levels of Meg3 in colonic cancer tissues from AOM/DSS-induced WT (+/+) and miR-708 KO (-/-) mice or from $A p c^{\min }$ WT $(+/+)$ mice and $A p c^{\min }$ miR-708 KO (-/-) mice. h RT-qPCR analyses were performed to detect the relative levels of Meg3 in organoids from $A p c^{\text {min }}$ WT $(+/+)$ mice or $A p c^{m i n}$ miR708 KO (-/-) mice. Organoids were treated with miR-708 agomir or miR708 antagomir and cultured for 6 days. i Representative images of organoids, derived from Apc $c^{\mathrm{min}}$ mice, after 6 days of culturing (scale bar: $100 \mu \mathrm{m}$ ). Meg3 interference adenovirus (Adv-Meg3i) or control adenovirus was administered to organoids that had been transfected with miR708 antagomir or control antagomir. j Representative images of organoids, derived from Apc ${ }^{\mathrm{min}}$ mice, after 8 days of culturing (scale bar: $100 \mu \mathrm{m})$. Meg3 overexpression adenovirus (Adv-Meg3) or control adenovirus was administered to organoids that had been transfected with miR-708 agomir or control agomir. The results are presented as the mean \pm S.D. in panels (b-j). NS not significant (non-paired two-tailed Student's $t$ test).

miR-708 promotes malignant proliferation of colonic cells via targeting of SOCS3 expression

Previous studies have suggested that SOCS3 inhibits CRC development and is indicative of a better prognosis in CRC patients $[28,29]$. Interestingly, bioinformatics analyses by several targeted mRNA-prediction tools have revealed the presence of miR-708-complementary sequences in the SOCS3 3'UTR; notably, these putative binding sequences are the same as those shared between Meg3 and miR-708 (Fig. 4a). Herein, we performed luciferase reporter assays to confirm the targeting relationship and found that expression of miR-708-WT led to a significant decrease in luciferase activity in CRC cell lines, whereas the luciferase activity in cells overexpressing miR-708-Mut was comparable to that in control cells (Fig. 4b). Consistently, forced expression of miR-708 was associated with decreased levels of SOCS3 mRNA and protein, while the introduction of a miR-708 antagomir led to the accumulation of SOCS3 mRNA and protein (Fig. 4c, e). In addition, a negative correlation between miR-708 and SOCS3 protein levels was observed in CRC cell lines (Fig. 4d).

Given that SOCS3 acts as a negative regulator of the JAK/STAT3 pathway [30, 31], we next assessed STAT3 activation. Western blotting and IF staining suggested that miR-708 overexpression elevates STAT3 activation in DLD1 cells, an observation that was further supported by the data from analyses of the expression of STAT3-targeted genes (Fig. 4e, Supplementary Fig. 5a, b). In addition, miR-708 deficiency led to attenuation of STAT3 activation via elevation of SOCS3 expression in colonic tissues from $A p c^{\mathrm{min}}$ mice as well as in colonic crypt cells from $A p c^{\min }$ mice (Fig. $4 \mathrm{f}, \mathrm{g}$ ). Consistent with those results, a miR-708 agomir inhibited SOCS3 accumulation, with associated potentiation of STAT3 activation in colonic organoids derived from crypts isolated from $A p C^{\text {min }}$ mice (Fig. 4g). Furthermore, IHC staining indicated that the level of p-STAT3 was decreased in AOM/DSS-induced miR-708 KO mice, in contrast to the effect in control mice (Supplementary Fig. 5c). Together, these data suggested that miR-708 activates STAT3 through antagonism of SOCS3 accumulation in CRC.

To further explore the effect of miR-708/SOCS3/STAT3 in the malignant proliferation of CRC cells, an adenovirus driving SOCS3 overexpression was used in both the 2D- and 3D-culturing systems. The results showed that SOCS3 overexpression blocked miR-708-fostered CRC cell proliferation in both culture systems (Fig. 4h, Supplementary Fig. 5d). Notably, the malignant proliferation of colonic crypt cells from $A p c^{\text {min }}$ mice with abundant miR-708 was markedly dampened by SOCS3; in contrast, SOCS3 knockdown reversed the inhibition of colonic organoid growth caused by loss of miR-708 in colonic crypts from $A p c^{\text {min }}$ mice (Fig. 4i).

Taken together, our results indicated that miR-708 promotes STAT3 activation by targeting the SOCS3 $3^{\prime}$-UTR, resulting in depletion of SOCS3 levels and causing malignant proliferation of colonic stem cells and CRC cell lines.

\section{Meg3 protects SOCS3 from miR-708-induced depletion}

Next, to determine the critical role of Meg3 in the miR-708/SOCS3 axis, we used luciferase reporter assays in RKO cells to show that Meg3 overexpression increased the luciferase activity of a SOCS3WT-containing construct; this effect was counteracted by the introduction of a miR-708 agomir (Fig. 5a). As expected, infection of DLD1 cells with the adenovirus-Meg3 interference construct resulted in a decrease in luciferase activity, which was restored by the introduction of a miR-708 antagomir (Supplementary Fig. 6a). qRT-PCR and western blot assays then were used to measure the potential regulation of SOCS3 expression by Meg3. The results showed that Meg3 overexpression enhanced SOCS3 mRNA and protein levels in RKO cells, while knockdown of Meg3 attenuated SOCS3 expression in DLD1 cells (Fig. 5b-d). However, the Meg3induced accumulation of SOCS3 was blocked by a miR-708 agomir (Fig. 5b, d). Furthermore, the expression of Meg3 and SOCS3 were analyzed in various CRC cell lines, revealing that Meg3 levels positively correlated with those of the SOCS3 protein (Supplementary Fig. 6b). Subsequently, activation of STAT3 was tested. The results suggested that Meg3 negatively regulated STAT3 activation and the expression of downstream target genes; those effects were reversed by a miR-708 agomir (Fig. 5b-d, Supplementary Fig. $6 c$, d). Thus, our data suggested that $M e g 3$ elevates SOCS3 levels by sponging miR-708 in CRC.

Functionally, the malignant proliferation of colonic stem cells was observed in the organoid culture system. Meg3i and SOCS3i adenoviruses were used to infect arganoids and in vitro cell culture (Supplementary Fig. 1g, h). We found that SOCS3 overexpression increased growth in Adv-Meg3i-infected organoids derived from the colonic crypts of $A p c^{\mathrm{min}}$ mice. In contrast, SOCS3 deficiency decreased the growth of these organoids when the cells were infected with Adv-Meg3 (Fig. 5e).

Together, these data revealed that Meg3-inhibited malignant proliferation in CRC is mediated by miR-708/SOCS3 signaling.

\section{Meg3/miR-708/SOCS3 pathway is associated with CRC development}

To further confirm the function of the Meg3/miR-708/SOCS3 axis in CRC development, we altered the expression of Meg3 and SOCS3, either alone or together, in the colons of $A p C^{\min } \mathrm{miR}_{-108^{-1-}}$ mice or $A p c^{\min }$ mice using adenovirus-mediated infection in vivo. We observed that Meg3 overexpression inhibited tumor growth in $A p c^{\min }$ mice, rendering a phenotype similar to that of $A p c^{\min } \mathrm{miR}^{-}$ $708^{-1-}$ mice (Fig. $6 \mathrm{a}-\mathrm{C}$ ). These results confirmed that Meg3 acts as a tumor suppressor in CRC, whereas miR-708 acts as an oncogene in CRC. However, the number and weight of tumors were increased in $A p C^{\text {min }}$ mice infected with Adv-SOCS3i + Adv-Meg3 (compared to $A p c^{\min }$ mice infected with control constructs). In addition, interference in SOCS3 expression in the colon promoted tumor growth in $A p C^{\min }$ miR-708 ${ }^{-1-}$ mice (Fig. 6a-C), indicating 
a

5' UGCAUGUGGGAAGUGGGCUCCUU 3' WT Meg3

3' GGGUCGAUCUAACAUUCGAGGAA 5' WT miR-708

5' ACAUCUUUCACCUCAGGCUCCUG 3' WT SOCS3 3' UTR

5' ACAUCUUUCACCUCAGCGAgGaG 3' mutant SOCS3 3' UTR

b
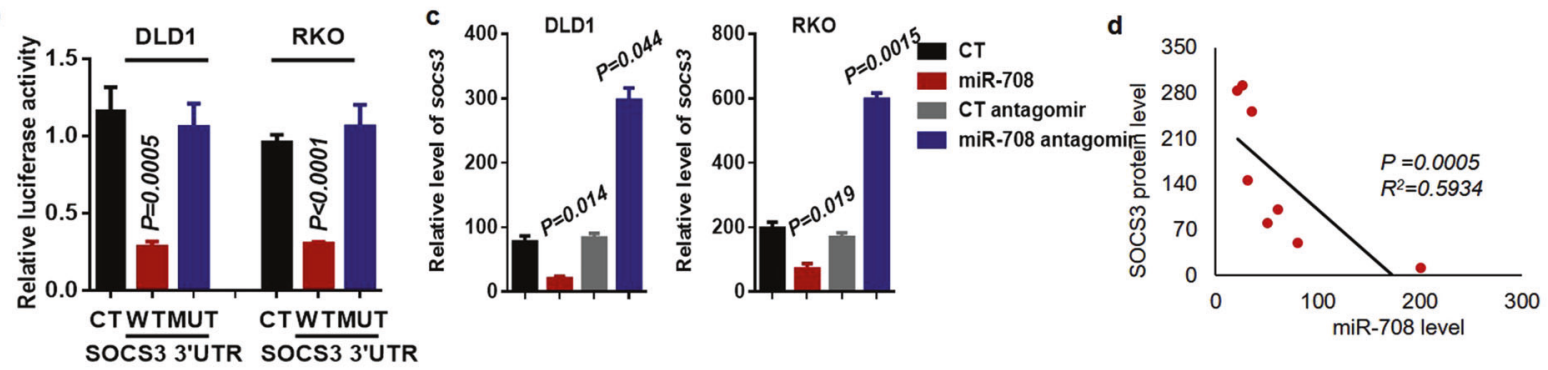

e
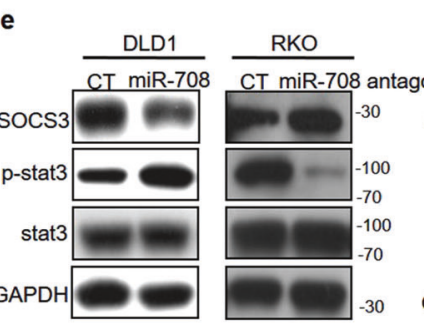

f

f $A P C^{\text {min }}$ miR-708 +/+ $\quad$ APC $^{+/ m i n}$ miR-708 - /-

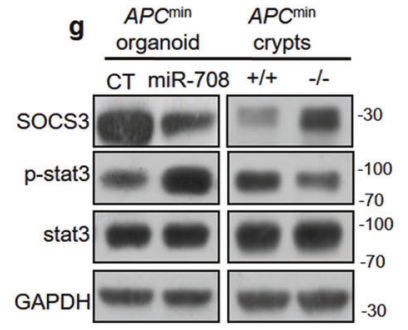

h
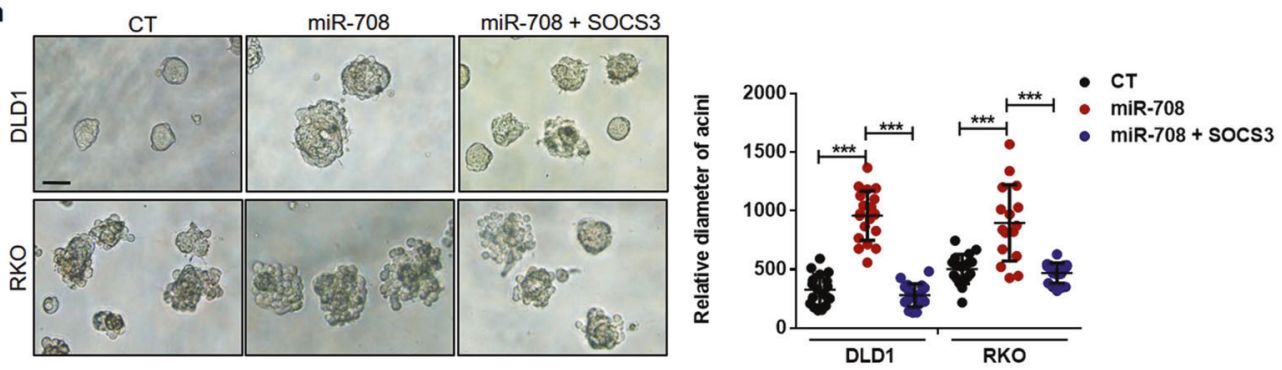

i
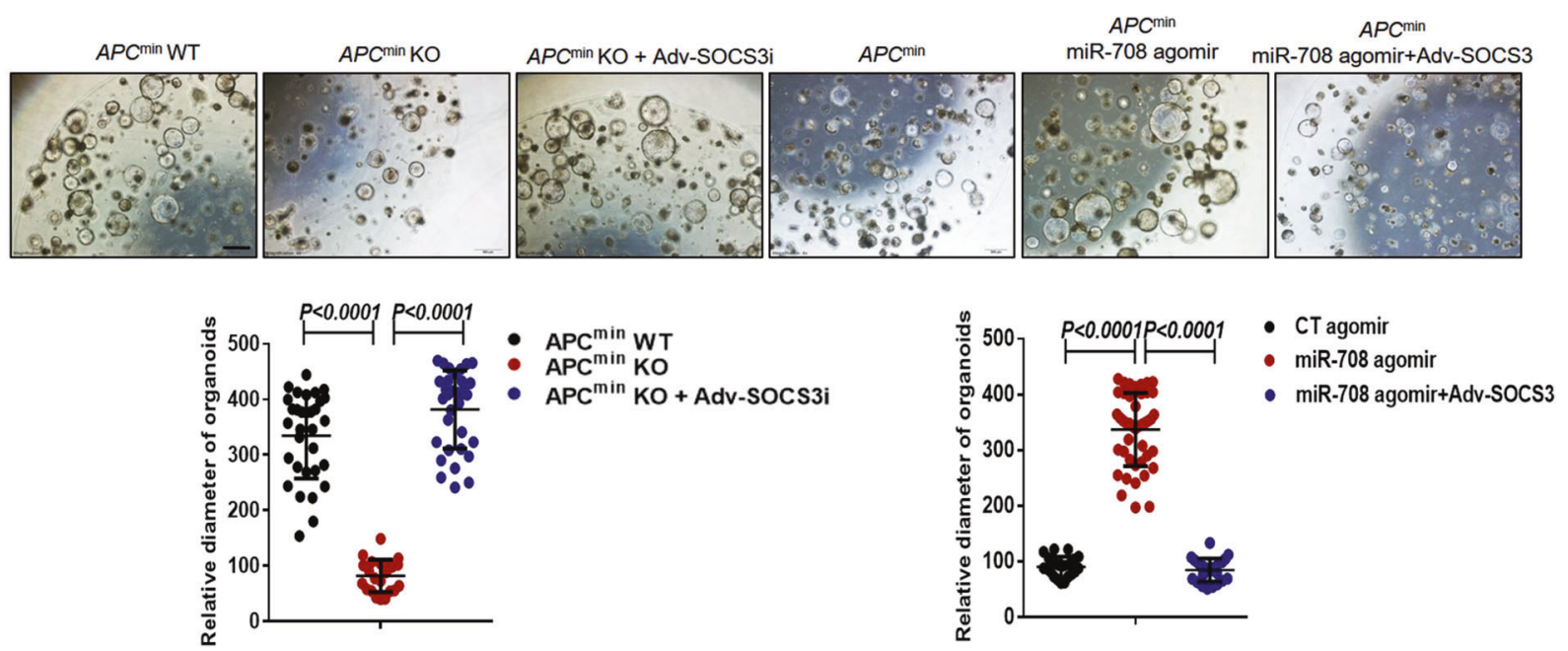

that the blocking effect of miR-708 deficiency on tumor growth was reversed by loss of SOCS3. Thus, SOCS3 is an effector of the Meg3/miR-708 signaling axis in vivo.

In further experiments, we cultured tumor organoids derived from CRC tissues of patients. We found that forced expression of Meg3 inhibited the growth of these organoids, while loss of SOCS3 counteracted the effect of Meg3 on organoid growth
(Fig. 6d). Subsequently, we examined the clinical significance of the findings established in our murine model. Analyses of the expression levels of Meg3, miR-708, and SOCS3 in human CRC tissues showed that Meg3 levels correlated positively with SOCS3 levels, while miR-708 levels correlated negatively with SOCS3 levels (Fig. 6e, f). Notably, these results in clinical samples paralleled the data obtained with CRC cell lines. 
Fig. 4 miR-708 promotes malignant proliferation of colonic cells via targeting of SOCS3 expression. a Predicted base-pairing between SOCS3 3'-UTR and miR-708. The mutant type (MUT) of the SOCS3 3'-UTR was generated by site-specific mutation of the paired bases. $\mathbf{b}$ The relative luciferase activities in DLD1 and RKO cells that were transfected with miR-708 and SOCS3 WT 3'UTR or SOCS3 MUT 3'UTR. c RT-qPCR analyses were performed to measure SOCS3 mRNA levels in DLD1 (left) and RKO (right) cells constitutively expressing miR-708, or in DLD1 and RKO cells transfected with a miR-708 antagomir. $\mathbf{d}$ The statistics of miR-708 levels and SOCS3 protein levels in eight colorectal cancer cell lines. e Western blotting was performed to detect levels of SOCS3 and activated STAT3 in RKO cells with knockdown of miR-708 and in DLD1 cells with forced expression of miR-708. $\mathbf{f}$ Western blotting was performed in colonic tumor tissues from Apc ${ }^{\min }$ WT mice and from Apc ${ }^{\text {min }} \mathrm{KO}^{\text {mice. }}$ g Western blotting was performed in organoids. Organoids derived from $A p c^{\text {min }}$ mice were treated with control agomir (CT) or miR-708 agomir (miR-708) and cultured for 6 days; organoids from $A p c^{\min }$ WT mice $(+/+)$ and $A p c^{\min }$ miR-708 KO mice $(-/-)$ were cultured for 6 days. h Representative images (scale bar: $100 \mu \mathrm{m}$ ) and quantification of the size of acinar sphere after 6 days of 3D-culture. Cells constitutively expressing miR-708 had been transfected with pCMV-SOCS3. i Representative images (scale bar: $100 \mu \mathrm{m}$ ) and quantification of the size of organoids from $A p c^{\mathrm{min}}$ mice. Organoids, derived from $A p c^{\mathrm{min}}$ WT mice and from $A p c^{\mathrm{min}}$ miR-708 KO mice, were infected with Adv-control or Adv-SOCS3 and cultured for 7 days; organoids derived from $A p c^{m i n}$ mice were treated with miR-708 agomir or control agomir and Adv-control or Adv-SOCS3 and were cultured for 7 days. The results are presented as the mean \pm S.D. in panels $(\mathbf{b}$, c, h, i). Statistical analyses were conducted by non-paired two-tailed Student's $t$ tests.

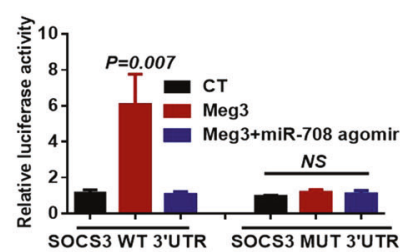

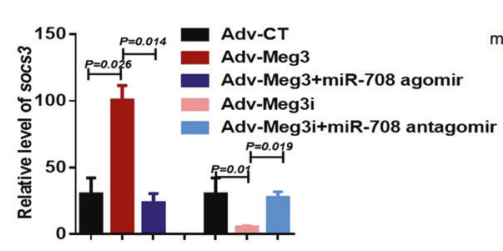

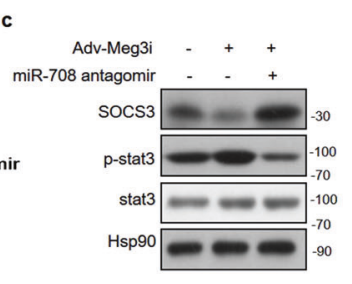

d

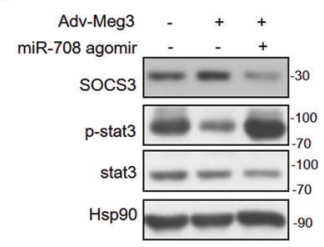

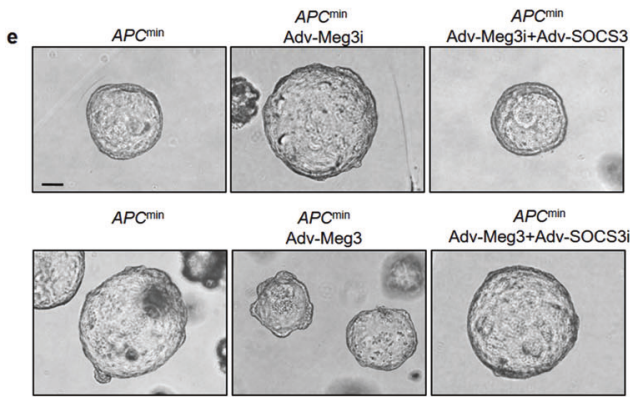
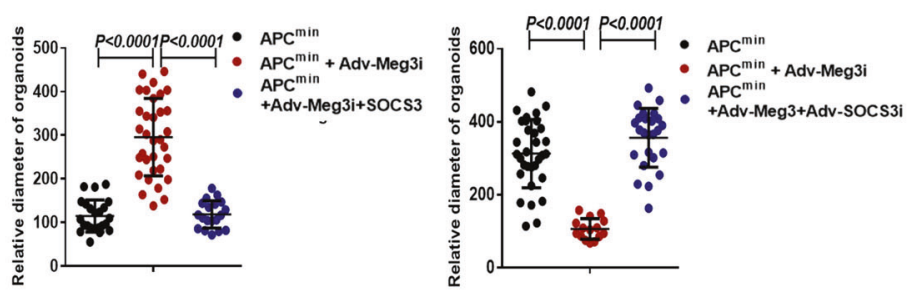

Fig. 5 Meg3 protects SOCS3 from miR-708-induced depletion. a The relative luciferase activities in RKO cells that had been transfected with indicated constructs. b Relative levels of Meg3 were detected by qPCR in RKO cells that had been infected with indicated adenovirus (to overexpress or knockdown Meg3) and then treated with miR-708 agomir or miR-708 antagomir, respectively. c Western blotting was performed to measure SOCS3 and activated STAT3 in DLD1 cells. Cells were infected with Adv-Meg3i, followed by treatment with miR-708 antagomir or control antagomir. d Western blotting was performed to measure SOCS3 and activated STAT3 in RKO cells. Cells were infected with Adv-Meg3, followed by treatment with miR-708 agomir or control agomir. e Representative images (scale bar: $100 \mu \mathrm{m}$ ) and quantification of organoid size. Upper: Organoids derived from $\mathrm{Apc}^{+/ \min }$ mice were infected with Adv-control, Adv-Meg3i, or Adv-Meg3i + Adv-SOCS3, or infected with Advcontrol, Adv-Meg3, or Adv-Meg3 + Adv-SOCS3i. The results are presented as the mean \pm S.D. in panels (a, b, and e). NS not significant (non-paired two-tailed Student's $t$ test).

Thus, the Meg3/miR-708/SOCS3 signaling axis is relevant in human CRC.

Collectively, these results demonstrated that the Meg3/miR-708/ SOCS3 signaling axis exists in CRC.

\section{DISCUSSION}

In the present study, we uncovered, for the first time (to our knowledge), the underlying mechanism by which Meg3 inhibits the malignant proliferation of colonic stem cells in early stage CRC. Meg3 levels negatively correlated with miR-708 levels, both in vivo and in vitro, particularly in colonic crypts from subjects with CRC. In addition, miR-708 deficiency attenuated colon tumor development and colonic organoid growth via direct targeting of SOCS3 accumulation. Importantly, Meg3 acts as a ceRNA for miR-708, leading to enhanced expression of SOCS3, which in turn suppresses STAT3 signaling and malignant proliferation of colonic stem cells during the early stage of colon tumor formation. In the clinic, the high miR-708 expression has been shown to indicate poor prognosis in patients with CRC.
Based on these data, we hypothesize that Meg3 functions as a ceRNA to regulate SCOS3 expression through sponging of miR708, thereby exerting regulatory functions to inhibit the malignant proliferation of colonic stem cells in early stage CRC. Thus, Meg3 may be a promising target for diagnosis and therapy of early stage CRC.

The IncRNA Meg3, which is abundantly expressed in many normal tissues, plays a central role in development and growth [32]. However, increasing evidence suggests that Meg3 frequently is either lost or depleted in multiple human tumors and tumor cell lines [11-13]. Clinically, Meg3 levels strongly correlated with the clinicopathological outcomes of various cancers [12]. In particular, lower levels of $\mathrm{Meg} 3$ are associated with worse pathological grade and advanced pathological TNM stage in CRC [14], an observation that is consistent with the results of the present study. Interestingly, our data revealed, for the first time, that Meg3 serves as a critical inhibitor of the malignant proliferation of colonic stem cells, the cell type that is mainly responsible for the majority of CRC development, especially in early stage CRC. Given the significant role of pathological stage at diagnosis in the clinic, 
a

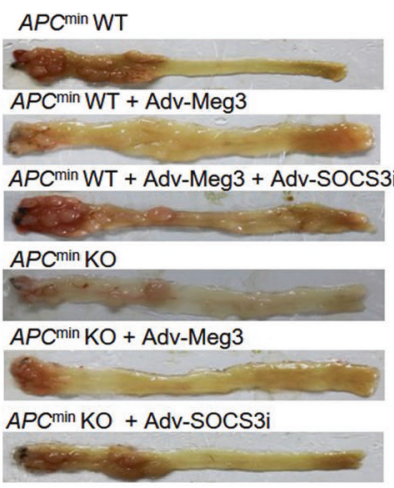

b

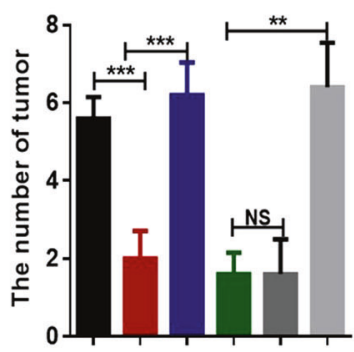

c

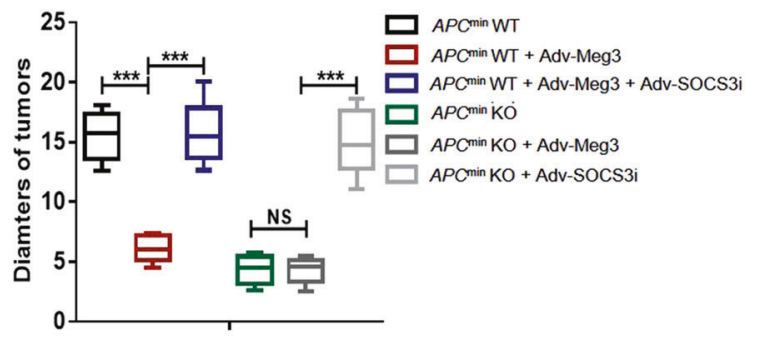

d

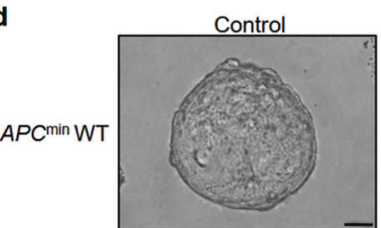

Control

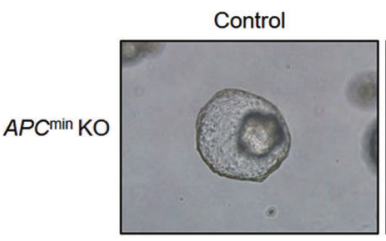

Adv-Meg3

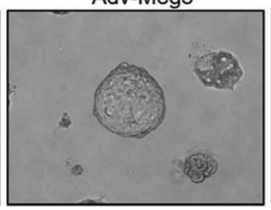

Adv-Meg3

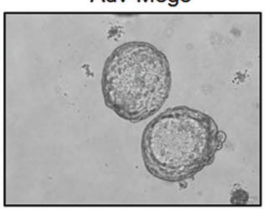

Adv-Meg3+Adv-SOCS3i

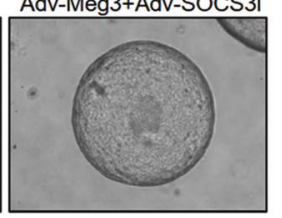

Adv-SOCS3i
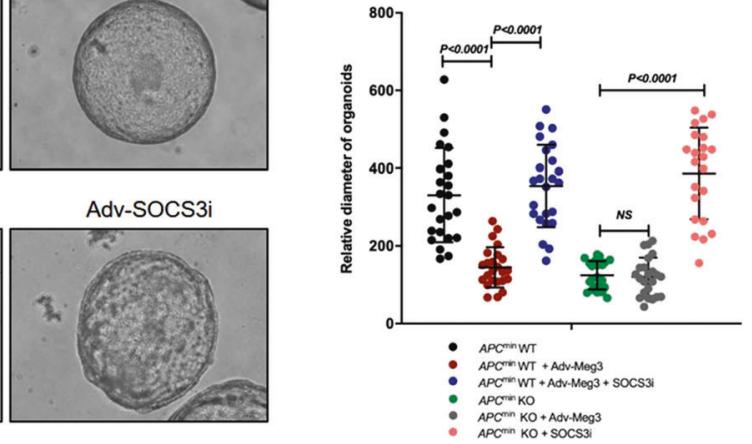

e
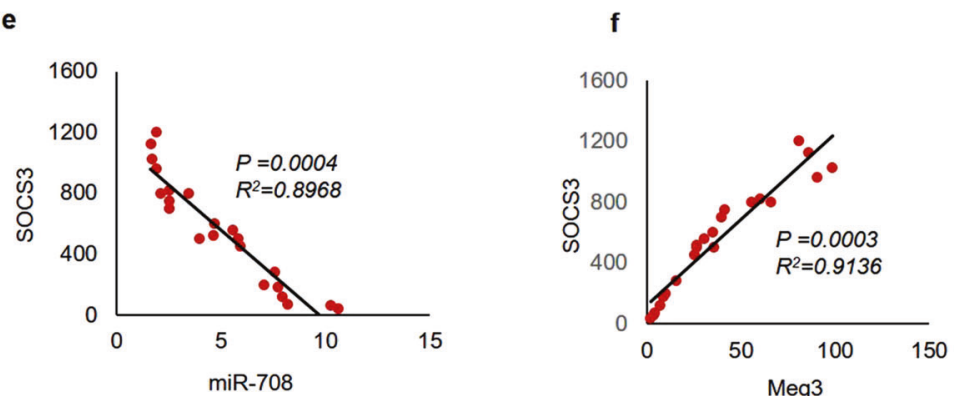

Fig. 6 The Meg3/miR-708/SOCS3 pathway is closely associated with CRC development. a-c $A p c^{\min }$ WT mice or $A p c^{\min }$ KO mice were infected with Adv-Meg3, Adv-SOCS3i, or Adv-Meg3 + Adv-SOCS3i by coloclysis. a Representative images of colonic tumors from Apc ${ }^{\text {min }}$ mice subjected to the indicated treatments. b Plot showing the numbers of tumors (diameter: $2-4 \mathrm{~mm}$ ) from $A p c^{\mathrm{min}}$ mice subjected to the indicated treatments. c The statistics of the diameters of tumors from $A p c^{\mathrm{min}}$ mice subjected to the indicated treatments. d Typical images (scale bar: $100 \mu \mathrm{m})$ of organoids derived from the mice presented in (a). e, f Plots showing the correlation between miR-708, Meg3, and SOCS3 levels in CRC tissues $(N=22)$. The results are presented as the mean \pm S.D. in panels (b and $\mathbf{c})$. NS not significant (non-paired two-tailed Student's $t$ test).

Meg3 might be a promising candidate for application in the diagnosis of early stage CRC.

IncRNA, by serving as ceRNAs, are known to regulate transcript accumulation at the post-transcriptional level by competing for miRNAs harboring complementary sequences [9]. Meg3 exhibits its biological roles via miRNAs that have been identified based on their roles in cancer, including (for example) miR-141 in chemotherapy [33], miR-212 in EMT [34], miR-19a and miR-93 in the cell cycle [35,36], and miR-21-5p in cell apoptosis [37]. In contrast, there have been (to our knowledge) only a limited number of studies related to the potential role of a Meg3/miRNA axis in CRC. In the present study, we revealed that Meg3 acts as ceRNA to sponge miR-708 in early stage CRC. Several studies have confirmed that miR-708 acts as an oncogenic miRNA via regulation of proliferation in CRC cell lines, as verified in the present work. Our results further showed that miR-708 accumulates to highgrade in tumor tissues from patients with CRC, such that the miR708 levels positively correlated with CRC progression. Importantly, we also showed, using mouse models and organoid culture systems, that miR-708 is responsible for the malignant proliferation of colonic stem cells and early stage CRC development. Notably, Meg3 deficiency dramatically attenuated tumor growth in $A p c^{m i n}$ miR- $708^{-1-}$ mice, hinting that the combined application of agomirs of Meg3 and antagomirs of miR-708 might contribute to therapy of CRC in the clinic.

Our analysis also demonstrated that regulation of mRNA accumulation is involved in events downstream of this IncRNA/ miRNA signaling axis. Our bioinformatics analyses and luciferase assays verified SOCS3 as a potential target mRNA of the Meg3/ miR-708 axis. As a critical negative regulator of the JAK/STAT signaling pathway, SOCS3 inhibits proliferation, migration, and invasion by blocking STAT3 activation, which is critical for lymph node metastasis and advanced clinical stages of CRC [28-31, 38]. Interestingly, our data indicated that manipulating miRNA-708 could influence the expression of Meg3. We found that quite a few studies reported that IL6 activates STAT3 to unregulated 
DNA methyltransferase (DNMT), such as DNMT3b and DNMT expression is important to the tumorigenesis [39-41]. It is known that DNA-demethylating drugs blocked cell proliferation and activated Meg3 expression. We have demonstrated the miRNA$708 /$ SOCS3/p-STAT3 axis in colon tumorigenesis in this study. We guess that miRNA-708 may upregulate Meg3 by evaluation of DNMT3b, which was related to inhibition of SOCS3 and activation of p-STAT3. The present study demonstrated that Meg3 elevates SOCS3 accumulation by sponging miR-708; the resulting increase in SOCS3 accumulation impedes STAT3 activation and finally dampens the malignant proliferation of colonic stem cells in early stage CRC. Intriguingly, previous data suggested that STAT3 regulates Meg3 expression by binding to the Meg3 promoter [42]. Thus, a feedback loop appears to exist between Meg3 and the SOCS3/STAT3 signaling pathway, an insight that deserves further exploration.

In conclusion, the present study demonstrated that Meg3 sponges miR-708 to inhibit early stage CRC development; this effect is mediated by SOCS3 inhibition of malignant proliferation of colonic stem cells. Thus, these results provide a novel insight into the mechanism underlying the role of $M e g 3$ in early stage CRC. Targeting of Meg3 and miR-708 might represent a promising strategy in both the diagnosis and therapy of colorectal tumors.

\section{DATA AVAILABILITY}

The datasets used and analyzed during the current study are available from the corresponding author on reasonable request.

\section{REFERENCES}

1. Weitz J, Koch M, Debus J, Höhler T, Galle PR, Büchler MW. Colorectal cancer. Lancet. 2005;365:153-65.

2. Dickinson BT, Kisiel J, Ahlquist DA, Grady WM. Molecular markers for colorectal cancer screening. Gut. 2015;64:1485-94.

3. Brenner H, Kloor M, Pox CP. Colorectal cancer. Lancet. 2014;383:1490-502.

4. Barker N. Adult intestinal stem cells: critical drivers of epithelial homeostasis and regeneration. Nat Rev Mol Cell Biol. 2014;15:19-33.

5. Zeuner A, Todaro M, Stassi G, De Maria R. Colorectal cancer stem cells: from the crypt to the clinic. Cell Stem Cell. 2014;15:692-705.

6. Schepers AG, Snippert HJ, Stange DE, Born M, Es JH, Wetering M, et al. Lineage tracing reveals Lgr5+ stem cell activity in mouse intestinal adenomas. Science. 2012;337:730-5.

7. Okugawa Y, Grady WM, Goel A. Epigenetic alterations in colorectal cancer: emerging biomarkers. Gastroenterology. 2015;149:1204-25.

8. Costa-Pinheiro P, Montezuma D, Henrique R, Jerónimo C. Diagnostic and prognostic epigenetic biomarkers in cancer. Epigenomics. 2015;7:1003-15.

9. Kita Y, Yonemori K, Osako Y, Baba K, Mori S, Maemura K, et al. Noncoding RNA and colorectal cancer: its epigenetic role. J Hum Genet. 2017;62:41-47.

10. Thomson DW, Dinger ME. Endogenous microRNA sponges: evidence and controversy. Nat Rev Genet. 2016;17:272-83.

11. Zhou Y, Zhang X, Klibanski A. MEG3 noncoding RNA: a tumor suppressor. J Mol Endocrinol. 2012;48:R45-53.

12. He Y, Luo Y, Liang B, Ye L, Lu G, He W. Potential applications of MEG3 in cancer diagnosis and prognosis. Oncotarget. 2017;8:73282-95.

13. Al-Rugeebah A, Alanazi M, Parine NR. MEG3: an oncogenic long non-coding RNA in different cancers. Pathol Oncol Res. 2019;25:859-74.

14. Yin DD, Liu ZJ, Zhang E, Kong R, Zhang ZH, Guo RH. Decreased expression of long noncoding RNA MEG3 affects cell proliferation and predicts a poor prognosis in patients with colorectal cancer. Tumour Biol. 2015;36:4851-9.

15. Zhu Y, Chen P, Gao Y, Ta N, Zhang Y, Cai J, et al. Meg3 activated by Vitamin D inhibits colorectal cancer cells proliferation and migration via regulating clusterin. EBioMedicine. 2018;30:148-57.

16. Li X, Ao J, Wu J. Systematic identification and comparison of expressed profiles of IncRNAs and circRNAs with associated co-expression and ceRNA networks in mouse germline stem cells. Oncotarget. 2017;8:26573-90.

17. Zhao Y, Zhu Z, Shi S, Wang J, Li N. Long non-coding RNA MEG3 regulates migration and invasion of lung cancer stem cells via miR-650/SLC34A2 axis. Biomed Pharmacother. 2019;120:109457.

18. De Robertis M, Massi E, Poeta ML, Carotti S, Morini S, Cecchetelli L, et al. The AOM/DSS murine model for the study of colon carcinogenesis: from pathways to diagnosis and therapy studies. J Carcinog. 2011;10:9.
19. Xu Y, Chang R, Peng Z, Wang Y, Ji W, Guo J, et al. Loss of polarity protein AF6 promotes pancreatic cancer metastasis by inducing Snail expression. Nat Commun. 2015;6:7184.

20. Wang N, Song L, Xu Y, Zhang L, Wu Y, Guo J, et al. Loss of Scribble confers cisplatin resistance during NSCLC chemotherapy via Nox2/ROS and Nrf2/PDL1 signaling. EBioMedicine. 2019;47:65-77.

21. Zhuang M, Zhao S, Jiang Z, Wang S, Sun P, Quan J, et al. MALAT1 sponges miR$106-5 p$ to promote the invasion and metastasis of colorectal cancer via SLAIN2 enhanced microtubules mobility. EBioMedicine. 2019;41:286-98.

22. Sansom OJ, Reed KR, Hayes AJ, Ireland H, Brinkmann H, Newton IP, et al. Loss of $A p c$ in vivo immediately perturbs Wnt signaling, differentiation, and migration. Genes Dev. 2004;18:1385-90.

23. Yamada Y, Mori H. Multistep carcinogenesis of the colon in $\mathrm{Apc}(\mathrm{Min} /+)$ mouse. Cancer Sci. 2007;98:6-10.

24. Monteleone NJ, Lutz CS. miR-708-5p: a microRNA with emerging roles in cancer. Oncotarget 2017;8:71292-316.

25. Lei SL, Zhao H, Yao HL, Chen Y, Lei ZD, Liu KJ, et al. Regulatory roles of microRNA708 and microRNA-31 in proliferation, apoptosis and invasion of colorectal cancer cells. Oncol Lett. 2014;8:1768-74.

26. Necela BM, Carr JM, Asmann YW, Thompson EA. Differential expression of microRNAs in tumors from chronically inflamed or genetic (APC(Min/+)) models of colon cancer. PLoS ONE. 2011;6:e18501.

27. Romero-Cordoba SL, Salido-Guadarrama I, Rodriguez-Dorantes M, HidalgoMiranda A. miRNA biogenesis: biological impact in the development of cancer. Cancer Biol Ther. 2014;15:1444-55.

28. Chu Q, Shen D, He L, Wang H, Liu C, Zhang W. Prognostic significance of SOCS3 and its biological function in colorectal cancer. Gene. 2017;627:114-22.

29. Li Y, de Haar C, Peppelenbosch MP, van der Woude CJ. SOCS3 in immune regulation of inflammatory bowel disease and inflammatory bowel disease-related cancer. Cytokine Growth Factor Rev. 2012;23:127-38.

30. Carow B, Rottenberg ME. SOCS3, a major regulator of infection and inflammation. Front Immunol. 2014;5:58.

31. Yin Y, Liu W, Dai Y. SOCS3 and its role in associated diseases. Hum Immunol. 2015;76:775-80.

32. da Rocha ST, Edwards CA, Ito M, Ogata $T$, Ferguson-Smith AC. Genomic imprinting at the mammalian Dlk1-Dio3 domain. Trends Genet. 2008;24:306-16.

33. Wang $\mathrm{H}$, Li H, Zhang L, Yang D. Overexpression of MEG3 sensitizes colorectal cancer cells to oxaliplatin through regulation of miR-141/PDCD4 axis. Biomed Pharmacother. 2018;106:1607-15.

34. Yu F, Geng W, Dong P, Huang Z, Zheng J. LncRNA-MEG3 inhibits activation of hepatic stellate cells through SMO protein and miR-212. Cell Death Dis. 2018;9:1014.

35. Qin N, Tong GF, Sun LW, Xu XL. Long noncoding RNA MEG3 suppresses glioma cell proliferation, migration, and invasion by acting as a competing endogenous RNA of miR-19a. Oncol Res. 2017;25:1471-8.

36. Zhang L, Liang $\mathrm{X}, \mathrm{Li} \mathrm{Y}$. Long non-coding RNA MEG3 inhibits cell growth of gliomas by targeting miR-93 and inactivating PI3K/AKT pathway. Oncol Rep. 2017;38:2408-16.

37. Zhang J, Yao T, Wang Y, Yu J, Liu Y, Lin Z. Long noncoding RNA MEG3 is downregulated in cervical cancer and affects cell proliferation and apoptosis by regulating miR-21. Cancer Biol Ther. 2016;17:104-13.

38. Vakil L, Najafipour R, Rakhshani N, Zamani F, Morakabati A, Javadi A. Investigation of FIH-1 and SOCS3 expression in KRAS mutant and wild-type patients with colorectal cancer. Tumour Biol. 2016;37:8841-8.

39. Liu CC, Lin JH, Hsu TW, Su K, Li AF, Hsu HS, et al. IL-6 enriched lung cancer stemlike cell population by inhibition of cell cycle regulators via DNMT1 upregulation. Int J Cancer. 2015;136:547-59.

40. Li Y, Deuring J, Peppelenbosch MP, Kuipers EJ, de Haar C, van der Woude CJ. IL-6induced DNMT1 activity mediates SOCS3 promoter hypermethylation in ulcerative colitis-related colorectal cancer. Carcinogenesis. 2012;33:1889-96.

41. Zhang Q, Wang HY, Marzec M, Raghunath PN, Nagasawa T, Wasik MA. STAT3- and DNA methyltransferase 1-mediated epigenetic silencing of SHP-1 tyrosine phosphatase tumor suppressor gene in malignant T lymphocytes. Proc Natl Acad Sci USA. 2005;102:6948-53.

42. Zhang J, Liang Y, Huang X, Guo X, Liu Y, Zhong J, et al. STAT3-induced upregulation of IncRNA MEG3 regulates the growth of cardiac hypertrophy through miR-361-5p/HDAC9 axis. Sci Rep. 2019;9:460.

\section{AUTHOR CONTRIBUTIONS}

S.Z. and X.H. planned and supervised the experimental work and data analysis; L.X.Z. and W.-W.J. performed all the experiments; W.W. and L.X.Z. analyzed and interpreted the data; X.H. and S.Z. wrote the paper. All authors discussed and revised the paper. 
FUNDING

This work was funded by grants from the National Key R\&D Program of China (2017YFC1600100), the National Natural Science Foundation of China (81673745 and 81902446), Traditional Chinese Medicine Science and Technology Key Program of Zhejiang Province of China (No. 2021ZZ013).

\section{COMPETING INTERESTS}

The authors declare no competing interests.

\section{ADDITIONAL INFORMATION}

Supplementary information The online version contains supplementary material available at https://doi.org/10.1038/s41419-021-04470-5.

Correspondence and requests for materials should be addressed to Xuan Huang.

Reprints and permission information is available at http://www.nature.com/ reprints
Publisher's note Springer Nature remains neutral with regard to jurisdictional claims in published maps and institutional affiliations.

(i) Open Access This article is licensed under a Creative Common Attribution 4.0 International License, which permits use, sharing adaptation, distribution and reproduction in any medium or format, as long as you give appropriate credit to the original author(s) and the source, provide a link to the Creative Commons license, and indicate if changes were made. The images or other third party material in this article are included in the article's Creative Commons license, unless indicated otherwise in a credit line to the material. If material is not included in the article's Creative Commons license and your intended use is not permitted by statutory regulation or exceeds the permitted use, you will need to obtain permission directly from the copyright holder. To view a copy of this license, visit http://creativecommons. org/licenses/by/4.0/.

(c) The Author(s) 2021 